\title{
Simulation study of first and second harmonic photocathode bimodal gun
}

\author{
Lin Wang (1) \\ Shanghai Institute of Applied Physics, Chinese Academy of Sciences, Shanghai 201800, China \\ University of Chinese Academy of Sciences, Beijing 100049, China \\ and Shanghai Synchrotron Radiation Facility, Shanghai 201204, China \\ Yong Jiang $\oplus^{*}$ \\ Wright Laboratory, Department of Physics, Yale University, New Haven, Connecticut 06520 USA \\ Wencheng Fang ${ }^{\dagger}$ and Zhentang Zhao \\ University of Chinese Academy of Sciences, Beijing 100049, China \\ Shanghai Advanced Research Institute, Chinese Academy of Sciences, Shanghai 201204, China \\ and Shanghai Synchrotron Radiation Facility, Shanghai 201204, China \\ Sergey V. Shchelkunov and Jay L. Hirshfield \\ Wright Laboratory, Department of Physics, Yale University, New Haven, Connecticut 06520 USA \\ and Omega-P R\&D Inc., New Haven, Connecticut 06511 USA
}

(Received 12 April 2020; accepted 21 January 2021; published 4 February 2021)

\begin{abstract}
The increasing demands for electron beams with high quality and low timing jitter for accelerator-based applications such as ultrafast electron microscopy (UEM) and ultrafast electron diffraction (UED) have stimulated active efforts to develop suitable injectors for these accelerators. One promising direction in this regard is using of a bimodal rf gun operating in fundamental mode and a phase-locked harmonically-related higher mode. Work reported in this paper is on analysis and design of a bimodal $r f$ gun operating in S-band as the fundamental and C-band as the second-harmonic modes. The rf design for such an of gun includes the new design like directional coupler and rectangular mode launcher, which presented good transmission, low reflective cross-talk, and allowed multiple iterations of the modification. Compared to the fundamental mode, the new design achieved reduction in rf emittance and energy spread for MeV UEM initial distribution, and reduction in slope of flight time as function of injection phase for MeV UED applications.
\end{abstract}

DOI: 10.1103/PhysRevAccelBeams.24.020401

\section{INTRODUCTION}

Accelerator-based electron beam applications like ultrafast electron diffraction (UED) and microscopy (UEM) have been proposed as promising tools to study substances at femtosecond and picosecond temporal scale [1]. Although the actual requirement and optimization for beam quality depend on the particular application, in all cases the ultimate performance of the application itself is essentially set by the lowest transverse emittance, the best control of the energy spread, or timing jitter allowed by the electron source. A number of radio frequency (rf) gun schemes,

\footnotetext{
*yong.jiang@aya.yale.edu

fangwencheng@zjlab.org.cn

zhaozhentang@sinap.ac.cn
}

Published by the American Physical Society under the terms of the Creative Commons Attribution 4.0 International license. Further distribution of this work must maintain attribution to the author(s) and the published article's title, journal citation, and DOI. based on single high frequency mode resonant cavities with photocathodes, have already demonstrated the required performance level to operate an UEM or UED, where notably emittance, energy spread are suppressed [2-4]. While successful, the pursuit for higher performance has never stopped.

The transverse emittance of rf gun is predominately determined by the thermal, space charge and rf emittances [5]. The thermal emittance depends upon the excess energy after photoemission and the initial beam size. The space charge emittance is caused by the self-field forces within the beam [6]. The rf emittance is the projected emittance due to the time-dependent rf defocusing within the gun. Electrons entering the $\mathrm{rf}$ field with different injection phases cause time-dependent rf transverse defocusing. A collection of slice phase-spaces with different slopes correspond to different time-dependent rf transverse defocusing. Thus, the projected rf transverse phase-space area is increased. The theory proposed by Kim [5] for single frequency rf guns, further developed by Serafini [7] and 
Zhao [8] for two-frequency rf guns, provides the basic analytic description of the rf emittance growth mechanisms in the two-frequency rf guns. By selecting a proper amplitude ratio and phase relationship between the fundamental and the harmonic rf field in the gun cavity, the superposition of the two frequencies can provide a temporally-flat-top-like rf profile, thus the electrons at the head and the tail of the electron bunch are influenced by similar rf kicks, decreasing the rf transverse emittance, the energy spread, and phase jitter. In response to this, a number of groups around the world started to study and develop alternative technologies and schemes that could generate low emittance beam using electron guns which are capable of operating at two harmonically-related frequencies [2,7-14].

The two-frequency rf gun configurations employing the first harmonic (S-band) plus a third harmonically related (X-band) $1^{\text {st }}+3^{\text {nd }}$ (S- and X-band) within a gun cavity to cancel the rf emittance were studied by $[8-12,14]$. The beam dynamic simulations based on the preliminary twofrequency cavity designs demonstrate advantages of the two-frequency gun over the single-frequency gun, including the generation of low transverse emittance electron beams with narrow RMS energy spectrum. Yet there are needs to explore critical technical problems. The twofrequency gun idea was never put into practice mainly because of overall technical complexity and cost compared to the other alternative schemes. The excessive reflection and leakage of the harmonic into the fundamental frequency klystron is also a hazard factor. Moreover, most of the simulations are based on free electron laser (FEL) application. In fact, UED and UEM are also the proper potential applications of bimodal gun. UEM now requires a further step forward in terms of low transverse emittance and energy spread. UED now requires a further step forward in terms of low timing jitter for higher temporal resolution [15].

In this paper, a bimodal gun is designed to apply microwaves at two harmonically-related frequencies 1 st and 2nd (S- and C-band) in a rf gun cavity. The frequency combination with lower harmonic frequency components like 1st and 2nd (S- + C-band), rather than 1st and 3rd (S- $+\mathrm{X}$-band), poses less budget for $\mathrm{rf}$ source, can achieve the similar beam dynamics benefits, since the 2nd harmonic C-band rf source are commercially available, but $9 \mathrm{GHz}$ $\mathrm{X}$-band klystron is not. The advanced 1st and 2nd ( $\mathrm{S}$ - and C-band) 0.6 cell bimodal electron gun was first proposed by researchers of Wright Laboratory, Department of Physics, Yale University [16]; and in the further collaboration with Shanghai Advanced Research Institute, Chinese Academy of Sciences [17]. In this paper, the further research design is being implemented to investigate the possibility of designing a relatively simple and costeffective bimodal gun that is capable of generating high quality beams with a small transverse emittance and low energy spread for potential application like UEM and low timing jitter for UED.
In Sec. II, the theory background of bimodal gun is described. Theories prove that the flat-top-like rf profile decreases the rf transverse emittance and the energy spread that are induced by the phase span of the electron bunch, and phase jitter cause by the curvature of field distribution. The deceleration effect caused by second harmonic is also pointed out in this section. In Sec. III, the rf design of the bimodal gun is presented. The rf design is capable of keeping good rf transmission, low reflectivity cross-talk, spurious TE mode suppression and allowing multiple iterations of the modification, which overcomes the challenges of the bimodal gun technical issues. In Sec. IV, the simulation results of the UED and UEM bunch initial distribution show benefits in the suppression of the rfinduced emittance, energy spread in UEM, and timing jitter in UED. As a consequence of these results, a final version of the presented bimodal gun is in the final fabrication stage at Shanghai Advanced Research Institute.

\section{CHARACTERISTICS OF THE BIMODAL GUN SCHEME}

Electrons generated at the cathode are accelerated by the rf field in the rf gun cavity. In the case of the fundamental mode excitation only, we assume that the rf electric field $E_{\text {fun }}$ along the axis of the cavity as a function of time $t$ and longitudinal coordinate $z$ can be roughly approximated as the following simple form:

$$
E_{\text {fun }}=E_{1} \cos (k z) \sin \left(\omega t+\phi_{10}\right)
$$

where $E_{1}$ is the $\mathrm{rf}$ accelerating field amplitude of the fundamental mode, $\phi_{10}$ is the 1 st harmonic rf phase as the particle leaves the cathode surface $z=0$ at $t=0, \lambda$ is the rf wavelength of the fundamental mode, $k=2 \pi / \lambda, c$ is the velocity of light, and $\omega=c k$. The field given by Eq. (1) can be considered to be produced by the rf cavity, bounded at one side by the cathode [18]. It is convenient to introduce the quantity of bunch exit phase $\phi$ :

$$
\phi=\omega t-k z+\phi_{10} .
$$

To understand the rf emittance and the energy spread caused by the longitudinal span of the electron bunch, $\phi$ in Eq. (2) is replaced by $\phi=\langle\phi\rangle+\delta \phi$, where $\langle\phi\rangle$ and $\delta \phi$ are the average exit phase and the phase of electrons in the bunch with respect to the reference particle of the electron bunch. After the acceleration of the rf gun cavity, the minimized rf emittance is as follows [5]:

$\varepsilon_{x, 11}^{r f}=\alpha_{0} k \frac{\left\langle x^{2}\right\rangle}{2} \sqrt{\left\langle(\delta \phi)^{4}\right\rangle-\left\langle(\delta \phi)^{2}\right\rangle^{2}} ; \quad\langle\phi\rangle=90^{\circ}$.

Here $\alpha_{0}=e E_{1} / 2 m_{0} c^{2} k$, a dimensionless parameter representing the strength of the accelerating field. As can be seen from Eq. (3), in this single mode gun, there is a 2 nd order 
term $\delta \phi^{2}$ of rf emittance caused by the span of the electron bunch. With $\langle\phi\rangle=90^{\circ}$, the energy spread caused by the span of the electron bunch $\delta \phi$ is

$\delta \gamma_{z, 11}=-\alpha_{0} \delta \phi-\frac{1}{2}\left(\gamma_{f, 11}-1\right)(\delta \phi)^{2}+\frac{\alpha}{3 !}(\delta \phi)^{3}+\cdots$

Here, $\gamma_{f, 11}$ is the final energy of the electron bunch. As shown in Eq. (4), there is high order phase dependence of energy spread.

In contrast to the single mode gun, the bimodal gun produces a flat-top of field in the cavity. The electron bunch is generated and transported in a flat-toplike field distribution which created by the two harmonically-related modes of which amplitudes and injection phases are matched. In the bimodal gun, the source of rf emittance can be eliminated by creating the flat-top effect.

According to Eq. (1), the harmonic rf electric field along the axis of the cavity as a function of time $t$ and longitudinal coordinate $z$ can be roughly approximated as:

$$
E_{\mathrm{har}}=E_{n} \cos (m k z) \sin \left(n \omega t+\phi_{n 0}\right)
$$

where $E_{n}$ is the rf accelerating field amplitude of the harmonic mode, $\phi_{n 0}$ is the harmonic rf phase as the particle leaves the cathode surface $z=0$ at $t=0$. The index $n$ is the harmonic number. If the frequency of the fundamental mode $f$ is $2856 \mathrm{MHz}, n=2$ is a harmonic mode $2 f$ of $5712 \mathrm{MHz}$, and $n=3$ is a harmonic mode $3 f$ of $8568 \mathrm{MHz}$. The index $m$ is the longitudinally spatial factor, indicating different longitudinal spatial modes. With different $m, E_{\text {har }}$ has different spatial period $2 \pi / m k$, and is independent of the harmonic number of frequency $n$. When a harmonic mode is added to the cavity, the on-axial electric field is composed of the fundamental mode and the harmonic mode. The total $\mathrm{rf}$ electric field along the axis of the cavity as a function of time $t$ and longitudinal coordinate $z$ can be roughly approximated as adding Eq. (5) to the main acceleration field Eq. (1):

$$
\begin{aligned}
E_{z, n m}= & E_{1} \cos (k z) \sin \left(\omega t+\phi_{10}\right) \\
& +E_{n} \cos (m k z) \sin \left(n \omega t+\phi_{n 0}\right) .
\end{aligned}
$$

In the bimodal operation, For the case $m=n=3$ [7] and the case $n=2, m=3$, with a proper amplitude and phase of harmonic mode and fundamental mode, the higher order of phase in energy spread can be canceled, and the minimum emittance can also be reduced. The above analysis, flat-top rf effect of bimodal gun indicates that the performance of the bimodal gun is quasi-DC, and the adverse impact of phase shift in bimodal gun is smaller than a single mode gun.

Several studies on bimodal gun in the last two decades [9-12] are all focused on the case $(m=n=3)$, demonstrating the prominent advantages of a bimodal $\mathrm{rf}$ gun over a single mode gun, especially the generation of low transverse emittance electron beams and narrow RMS energy spectrum. Yet there are needs to explore critical technical problems. The power delivering system for the two frequencies enhances the complexity of rf design, including coupler design and two rf systems. The other development bottleneck of bimodal gun is its higher cost of a extra X-band klystron. S/C ( $n=2, m=3$ case $)$ compared to $\mathrm{S} / \mathrm{X}[(m=n=3)$ case $]$ is better, due to the possibly easier rf design, and commercially available C-band klystron. In this paper, this scheme includes the combination of the harmonic mode parameter $n=2$, $m=3$, which simplifies fabrication while enhancing the beam quality. A compact, simple, and budget-friendly scheme is presented to realize the high order terms cancellation in the rf induced emittance and energy spread.

To demonstrate the beam dynamic benefits of $n=2$, $m=3$ case, the specific layout we present later in this paper combines the fundamental $\mathrm{TM}_{010}$ mode at the frequency of $2856 \mathrm{MHz}$, and the harmonic $\mathrm{TM}_{011}$ mode at the frequency of $5712 \mathrm{MHz}$. The CST [19] simulation result of $\mathrm{rf}$ field distribution in the half cell cavity is depicted in Fig. 1. This new harmonic choice opens up a variety of frequency range possibilities in bimodal $\mathrm{rf}$ guns supported by available high-power klystrons such as $\mathrm{L}+\mathrm{S}$-band, $\mathrm{S}+\mathrm{C}$-band, $\mathrm{C}+\mathrm{X}$-band, etc.

It should be clarified that, in the case $n=2, m=3$, the phase velocity of the harmonic mode equals $2 / 3 \mathrm{c}$, which is much slower than electron. The rf power for harmonic mode is wasted with little acceleration or deceleration. In this paper, the demonstrated simulation is based on half cell. For longer acceleration structure like 1.5 cell, the effect of beam-rf phase slippage is accumulated.

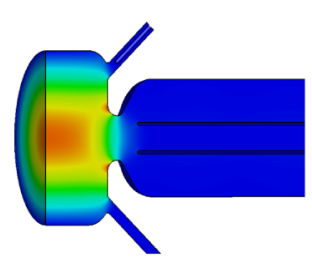

(a)

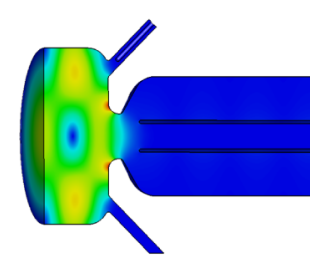

(b)

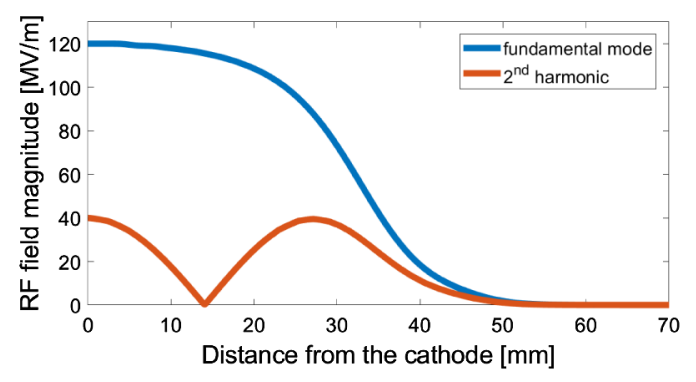

(c)
FIG. 1. The electric field distribution of the both modes in CST simulation. (a) The fundamental $\mathrm{TM}_{010}$ mode at $2856 \mathrm{MHz}$. (b) The harmonic $\mathrm{TM}_{011}$ mode at $5712 \mathrm{MHz}$. (c) The on-axial $E_{z}$ distribution of fundamental mode and 2 nd harmonic mode. 
To estimate the effect of beam-rf phase slippage in a $n=2$, $m=3$ case 1.5 bimodal gun, assuming $E_{n}$ and $E_{1}$ are $50 \mathrm{MV} / \mathrm{m}$ and $120 \mathrm{MV} / \mathrm{m}$, and the velocity of electron bunch is ultrarelativistic, the integration of Eq. (6) from $z=0$ to the exit of the 1.5 cell gun shows that the harmonic-mode-induced energy loss over the energy gain of single mode gun is $\sim 10 \%$. For applications such as UED and UEM, which do not require high energy electrons, $10 \%$ smaller energy is within allowance. The advantage and the beam deceleration of the 1.5-cell bimodal gun will be investigated from the beam dynamics point of view in a future study.

\section{III. rf DESIGN}

To utilize the bimodal gun advantages in modeling, compared to the single mode rf guns, coupling two resonant modes in a same gun is challenging. The design of the bimodal gun requires good transmission, low reflective cross-talk, and allowing multiple iterations of the modification. L. Serafini proposed an ingenious scheme [7] in principle for the coupling of the two resonant modes in the same cavity via two rectangular waveguides, which are placed with the gun cavity side-by-side. The only imperfection of this scheme is that there are potential problems of exciting higher order mode and harmonic mode leaking which cause damage to the fundamental power source.

To overcome these design issues, several novel design features are introduced in our new scheme, including: a twofrequency waveguide feed devised for low reflective crosstalk that could damage fundamental mode power source, a coaxial rf coupling and a mode launcher for multipole mode suppressing. This bimodal design includes high degrees of modularity to mitigate challenges in optimizing performance of each module of the gun assembly, allowing multiple iterations of the modification, and if necessary, improvement or replacement of each module.

\section{A. Bimodal gun 0.6-cell cavity}

The cavity body length is about $0.3 \lambda$ ( 0.6 cell). In a typical half-cell with sinusoidal temporal profile of cathode field, a longer first cell leads to operating injection phase shifted further away from the crest of the rf field. This will lower the initial rf field strength causing the electron bunch to suffer greater space charge forces during and just after emission [20].

The 3D design of the bimodal gun was accomplished using the code Computer Simulation Technology (CST) [19]. The geometry and the rf parameters of the 0.6 cell cavity are listed in Table I. The cavity length is based on the 0.6 cell of $2856 \mathrm{MHz}$, accommodating the S-band $\mathrm{TM}_{010}$ and $\mathrm{C}$-band $\mathrm{TM}_{011}$ simultaneously by adjusting the cavity diameter and the radius of chamber on the cavity. Assuming an arrangement of $120 \mathrm{MV} / \mathrm{m}$ amplitude S-band and $40 \mathrm{MV} / \mathrm{m}$ amplitude C-band, the required input power
TABLE I. rf parameters of the 0.6 cell cavity.

\begin{tabular}{lccc}
\hline \hline Parameter & Unit & S-band & C-band \\
\hline Cavity length & $\mathrm{mm}$ & 31.39 & 31.39 \\
Cavity diameter & $\mathrm{mm}$ & 82.58 & 82.58 \\
Iris aperture & $\mathrm{mm}$ & 21 & 21 \\
Frequency & $\mathrm{MHz}$ & 2856 & 5712 \\
E-field amplitude & $\mathrm{MV} / \mathrm{m}$ & 120 & 40 \\
$E_{\text {surfacepeak }} / E_{\text {amplitude }}$ & 1 & 1.1 & 1.55 \\
Required input power & $\mathrm{MW}$ & 2.4 & 1.6 \\
Coupling factor & 1 & 1.05 & 2.04 \\
Quality factor & $10^{4}$ & 1.4941 & 1.3840 \\
\hline \hline
\end{tabular}

will be $2.4 \mathrm{MW}$ for S-band, and 1.6 MW for C-band. The electric field distribution of the both modes in CST simulation are shown in Fig. 1.

\section{B. Assembly of waveguides and S-parameters}

The waveguide system consists of the assembly of the directional coupler and the mode launcher, as shown in Fig. 2. The port 1 is the S-band power input, the port 2 is the $\mathrm{C}$-band power input, and the port 3 is the combined output of the S-band and C-band, connecting to the bimodal gun cavity. The S-band excitation field distribution is shown in Fig. 2(a), and the C-band excitation field distribution is shown in Fig. 2(b).

The compact four-branch structure connecting the S-band and C-band rectangular waveguide is the directional coupler that combine S-band and C-band power, and further discussion of the directional coupler is in Sec. III C. The high directionality of the directional coupler guarantees the C-band of higher frequency is transported to the combined output without leaking into the S-band rectangular waveguide of lower cutoff frequency. The boxlike structure that connects the output of the directional coupler and the coaxial waveguide feeding the bimodal gun cavity is the mode launcher, converting the TE mode in the rectangular waveguide to the TEM mode in the coaxial waveguide. The special design of the mode launcher described in Sec. III D guarantees the suppression of the higher order spatial mode that caused by the single-side coupling.

The S-parameters in Fig. 2(c) and Fig. 2(d) precisely describe the transmission, reflection, and directionality performance of the desired $\mathrm{rf}$ mode in the waveguide system. The $S_{1(1), 2(1)}$ and $S_{1(1), 3(1)}$ in Fig. 2(d) show the directionality of the directional coupler. At the operating frequency of $5712 \mathrm{MHz}, S_{1(1), 2(1)}$ and $S_{1(1), 3(1)}$ are less than $-40 \mathrm{~dB}$, indicating that less than $0.01 \%$ of the C-band power transports into the S-band power source. At the coaxial waveguide output of port 3 , the asymmetry of single side coupling from rectangular waveguide to the coaxial waveguide causes undesired higher order spatial mode such as dipole TE mode, causing a beam quality 


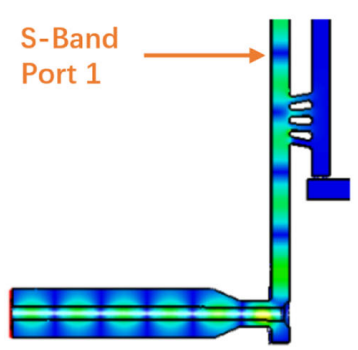

(a)

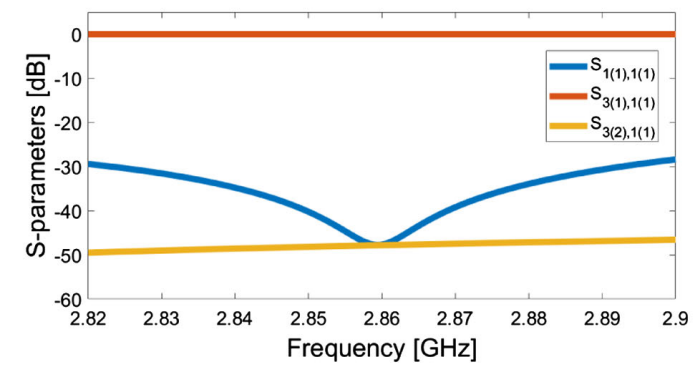

(c)

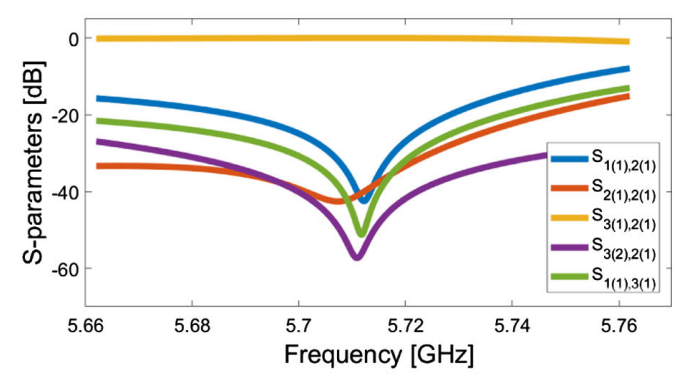

(d)

FIG. 2. The contour map of the electrical field distribution of the waveguide assembly and S-parameters. (a) Excited by S-band (b) Excited by C-band (c) S-parameters of the assembly excited by S-band (d) S-parameters of the assembly excited by C-band.

deterioration [21]. The ideal spatial mode is TEM mode, exciting the symmetrical rf field within the cavity. With special design, $S_{3(2), 1(1)}$ and $S_{3(2), 2(1)}$, the transmissions of the undesired dipole mode from port 1 to port 3 , and port 2 to port 3 are able to be suppressed to below $-50 \mathrm{~dB}$.

The transmissions of the desired TEM mode, $S_{3(1), 1(1)}$ and $S_{3(1), 2(1)}$, are better than $-0.001 \mathrm{~dB}$. There are transmission S-parameters for higher order asymmetrical modes like $S_{3(3), 2(1)}, S_{3(3), 1(1)}$ that are not plotted in the S-parameter chart, which are far lower than $-60 \mathrm{~dB}$. The $S_{1(1), 1(1)}$ in Fig. 2(c) and $S_{2(1), 2(1)}$ in Fig. 2(d) represent for the reflection of the S-band and C-band power input, which are all below $-40 \mathrm{~dB}$ at the operating frequencies. It is worth pointing out that the bandwidth of unwanted reflection or cross-talk that below $-30 \mathrm{~dB}$ are wilder than $10 \mathrm{MHz}$, which added more tolerance for the machining. Thus, the directional coupler scheme is not only of highperformance, but also feasible practically.

\section{Directional coupler}

A novel four branch directional coupler is described in this section, as inspired from the theory of the multiple branch waveguide coupler, which is developed for single frequency operation [22].

Adjustments are made to adapt the ideal multiple branch waveguide model for our two-frequencies operation and practical high-power application. Since the two rectangular waveguides have different dimensions and guide wavelength, the distances between branches are optimized to guarantee the $0 \mathrm{~dB}$ transfer. As can be seen in Fig. 3(a), the distances between the branches located on the S-band waveguide is optimized to be shorter than the one on the $\mathrm{C}$-band waveguide. The port 4 in the ideal multiple branch waveguide modal is been terminated by the vacuum port for bimodal application. The vacuum port is simulated as a group of cylinders and a cubic outlet in the lower right of the Fig. 3(a). To assure the directionality remains intact, the distance between the terminated wall and the branches is optimized, and bump structures are added near the port 2 to damp the reflection of the C-band power. Moreover, to produce a smoother surface within the directional coupler avoiding $\mathrm{rf}$ breakdown, rounding at the sharp corners of the branched is added. The optimized directional coupler field distribution is shown in Fig. 3. With an appropriate design of the dimensions, the S-band and C-band transmit directly into the combined output, and no obvious reflection and cross-talk are observed, realizing the $0 \mathrm{~dB}$ coupling as the principle model depicted.

\section{Mode launcher}

The geometry of the mode launcher with detail is shown in Fig. 4. To lower the manufacturing cost and to save space for more flexibility, single side coupling is applied in this design. Different from the traditional two-arm structure, the arms are confined in a box shape, which could save more space and be easier to fabricate. In the launcher box shown in Fig. 4(b), the one side input is divided into two by the

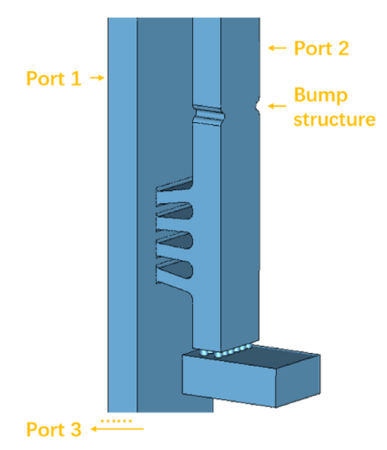

(a)

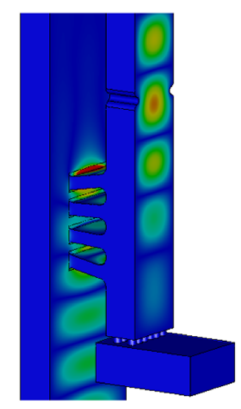

(b)
FIG. 3. The principle multibranch directional coupler model, and electrical field distribution in the optimized $0 \mathrm{~dB}$ directional coupler. (a) Excited by S-band (b) Excited by C-band. 


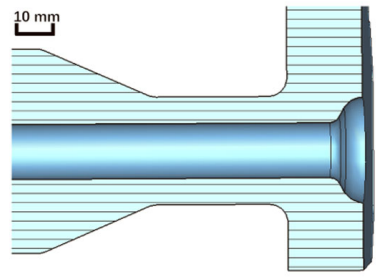

(a)

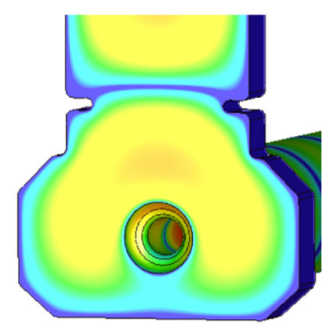

(c)

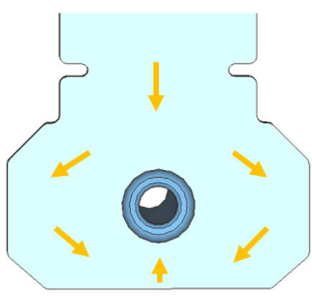

(b)

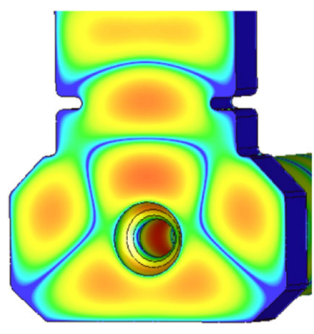

(d)
FIG. 4. The geometry of the mode launcher with detail, and the field distribution within the mode launcher once excited. (a) Cross section (b) Launcher box (c) Excited by S-band (d) Excited by C-band.

hole in the center of the launcher box. When the two feeds meet each other on the opposite side of the original input, the combined two feeds and the latter envelope form a symmetric field distribution at the entrance of the coaxial waveguide, similar to the traditional double-feed coupler pattern. The entrance of the coaxial waveguide is the narrow necklike cylinder in the middle of Fig. 4(a). The electrical field distribution in mode launcher shown in Fig. 4, depicts more detail of how the wave envelope divides and combines in the mode launcher.

To assure a good transmission of both S-band and C-band power, more degrees of freedom are added to the mode launcher. The four corners of the rectangular launcher box are chamfered. The narrow necklike cylinder that connects the box and the coaxial waveguide, is also acts as a filter that damps the spurious TE mode that the single-side coupling excited. After the optimization of the dimensions of the mode launcher, as can be seen in Fig. 2(a) and Fig. 2(b), the output field distribution within the coaxial waveguide is symmetrical, similar to the distribution of TEM mode.

\section{BEAM DYNAMICS SIMULATION IN BIMODAL GUN}

To demonstrate the bimodal gun concept, a bimodal rf gun together with a compensation coil have been modeled. The simulation results presented here are for the bimodal operation of the half-cell gun operating at $2856 \mathrm{MHz}$ and $5712 \mathrm{MHz}$. The injection phases and the field strengths of rf and peak field of compensation coil are optimized to minimize the normalized RMS transverse emittance.
The simulations are carried out with ASTRA [23]. To demonstrate the advantages of the bimodal gun in lowering transverse emittance, energy spread and timing jitter, the listed simulation results includes different initial charge, beam size and duration for ultrafast electron difference (UED) and ultrafast electron microscopy (UEM).

\section{A. UEM application}

The first study of $\mathrm{MeV}$ accelerator-based ultrafast electron microscope (UEM) [2] reveals the feasibility of rf photoinjector producing high quality images with nanoscale fine structures using a single bunch that contains millions of electrons (single-shot) with simultaneous picosecond temporal resolution and nanometer spatial resolution. So far, the only test of MeV UEM [3] records the imagining with pulsed $\sim 3 \mathrm{MeV}$ picosecond electron beam. The temporal and spatial resolution operating in single-shot mode is about 4 ps (FWHM) and $100 \mathrm{~nm}$ (FWHM). According to [2,3], if transverse emittance and energy spread are lower, there is still room for a higher spatial resolution. Hence, the researches of bimodal gun UEM application are carried out on field-emission cathode [10] and on photocathode $[2,13]$. Compared to field-emission cathode, photocathode rf guns [24,25] produce very highquality electron beams because the laser allows electron emission to be gated to a specific portion of the rf period. To avoid the complexity of the bimodal rf system, one of the creative solutions is to spatially separate the two resonate mode $f_{1}$ and $f_{2}$ into two cavities [2,13]. The only disadvantage of this approach is dilution of beam emittance when traveling in the fundamental mode gun and the drift pipe between the two cavities, which is irreversible.

Here, the simulated UEM initial beam distribution is as follows: the $6 \mathrm{pC}$ electron bunch has a RMS spot size of $20 \mu \mathrm{m}$ and a time duration of $10 \mathrm{ps}$ (FWHM). According to analysis in Sec. II, a wider longitudinal electron bunch span $\delta \phi$ causes a bigger rf emittance. Since the bimodal gun cancels the rf emittance, a relatively long bunch distribution underscores the advantages of bimodal mode. In order to control the beam envelope, a solenoid is located close to the cathode. It provides a focusing, and it also contributes to a reduction of the correlated emittance via the so-called emittance compensation process at the same time [26]. The solenoid on-axis field in POSSION [27] is shown in Fig. 5. The design of the solenoid composed by a main coil and a bucking coil [16], in order to get a vanishing solenoidal field at the cathode $(z=0)$, which would otherwise introduce an undesired emittance contribution [28]. To reduce input parameters and gain an overview of the beam dynamic in bimodal gun, the apertures and extra solenoids in experimental case are not added to this simulation.

The amplitude of S-band in single mode gun and bimodal gun are $120 \mathrm{MV} / \mathrm{m}$. The optimized lowest emittance nominal injection phase for single mode gun is $207^{\circ}$. The optimized lowest emittance nominal injection phase 


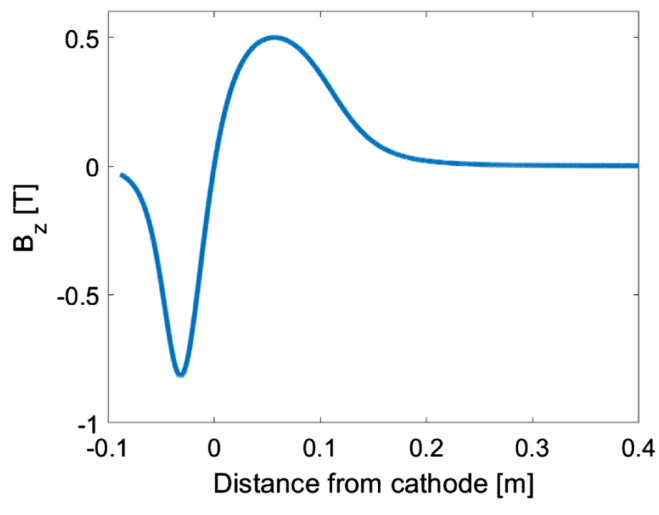

FIG. 5. $\quad B_{z}$ component of the solenoid on-axis field.

for bimodal gun mode gun is $230^{\circ}$ and $10^{\circ}$ for S-band and C-band. The amplitude of C-band is $50 \mathrm{MV} / \mathrm{m}$. The corresponding results are in Fig. 6. At 0.4 meters downstream of the cathode (the gun exit), the bunch size of the beam is shown in Fig. 6(b). The total transverse emittance of bimodal gun is reduced by $70 \%$, as shown in Fig. 6(c). The energy gain evolution along the distance from the cathode is as shown in Fig. 6(a). The RMS energy spread evolution along the distance from the cathode is as shown in Fig. 6(d). At the exit of the gun, the total energy spread of bimodal mode is 0.005 , while the single mode is 0.01 . Comparing to the single mode, the energy spread is reduced by $50 \%$ with bimodal mode, which can also improve the spatial resolution.

By placing fundamental mode and 2nd harmonic in the same cavity, the rf transverse emittance and energy spread are reduced, without the irreversible beam quality dilution as in the fundamental mode gun and the drift between the two cavities as in $[2,13]$.

\section{B. UED application}

In MeV UED [4], one of the most potential methods of visualization of atomic dynamics, the sample is driven to a nonequilibrium state by a laser, and then probed by a delayed electron pulse produced in a photocathode if gun. The pulse width and flight time jitter of the electron beam primarily determine the temporal resolution in MeV UED. Most recently, in a novel scheme [29], the electron beam produced in a photocathode rf gun first expands under its own Coulomb force, and is then sent through a double bend achromat (DBA), leading to longitudinal pulse compression. The DBA compression cancels the amplitude jitter that the previous compression methods such as rf buncher and laser-driven $\mathrm{THz}$ buncher produce. The only drawback here is the phase jitter in the DBA compression, which remain uncompensated. In contrast to the single mode gun, the bimodal gun produces a flat-top rf field in the cavity that the electron bunch is generated and transported. The two harmonically-related modes of which amplitudes and injection phases are matched create a flat-toplike field

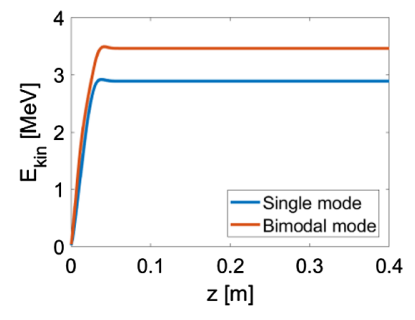

(a)

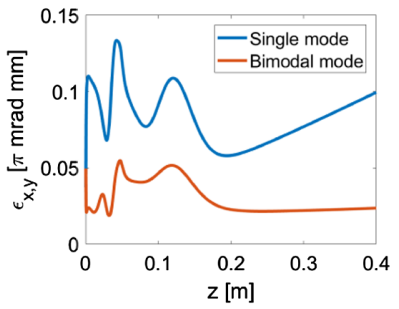

(c)

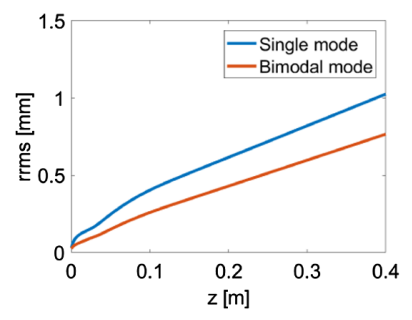

(b)

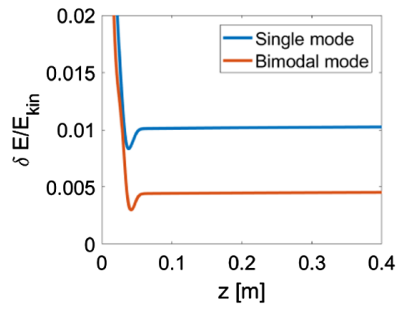

(d)
FIG. 6. Emittance compensation solenoid and beam dynamic performance along the $\mathrm{z}$ axis. (a) Energy gain evolution (b) Bunch size evolution (c) Transverse emittance evolution (d) RMS Energy spread evolution.

distribution. The theory of bimodal gun indicates that the performance of the bimodal gun is quasi-DC, and the adverse impact of phase shift in bimodal gun is smaller than a single mode gun.

In a rf gun, when the slope of flight time as function of the injection phase $d t / d \phi_{10}$ is close to zero, the adverse effect of phase jitter is minimized, since the flight time is impacted less by the injection phase shift. The characteristic of an ideal gun for low timing jitter MeV UED is minimizeing the timing jitter while maximizing the energy gain. To compare the performance on phase jitter of single mode gun and bimodal gun, simulations of different rfinjection-phases-related flight time and energy gain in both guns are carried out. The simulated UED initial beam distribution is as follows: the $200 \mathrm{fC}$ electron bunch has a RMS spot size of $0.04 \mathrm{~mm}$ and a time duration of $100 \mathrm{fs}$ (FWHM). The gradient of S-band and C-band are $120 \mathrm{MV} / \mathrm{m}$ and $40 \mathrm{MV} / \mathrm{m}$. The beamline is $0.4 \mathrm{~m}$ after the cathode. The nominal injection phase for maximum energy gain for single mode gun is S-band $190^{\circ}$. The nominal injection phases for maximum energy gain for bimodal gun are S-band $200^{\circ}$ and C-band $50^{\circ}$.

Figure 7(a) shows the slope of flight time as function of S-band injection phase $d t / d \phi_{10}$ in a $30^{\circ}$ range around the maximum energy gain. $\triangle \phi_{10}$ represents the S-band nominal injection phase distance away from the maximum energy gain. The point of $\triangle \phi_{10}=0$ represents the maximum energy gain. At the same phase distance away from the maximum energy gain nominal phase $\Delta \phi_{10}=0$, within a range of $\sim 30^{\circ}$, the slope of flight time as function of injection phase $d t / d \phi_{10}$ in bimodal gun is $\sim 60 \%$ lower than that of single mode gun, indicating that at the 


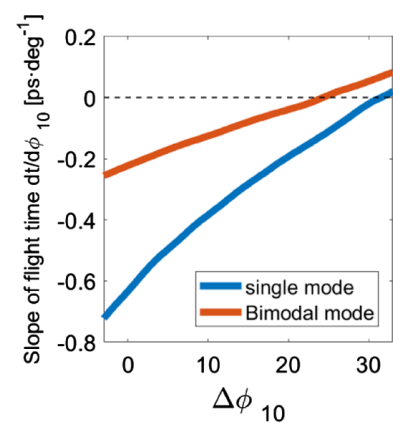

(a)

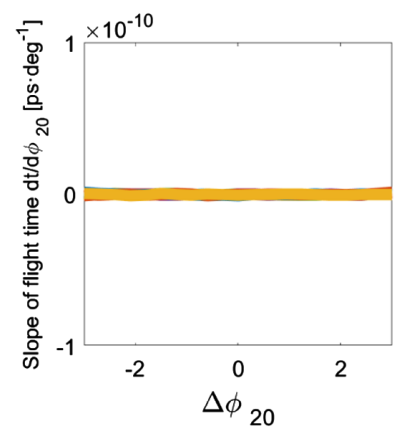

(b)
FIG. 7. Slope of flight time as function of injection phases at the gun exit. (a) The slope of ight time as function of fundamental mode injection phase $d t / d \phi_{10} . \Delta \phi_{10}^{\circ}=0$ represents the phase of maximum energy gain. (b) The slope of ight time as function of harmonic mode injection phase $d t / d \phi_{20} . \Delta \phi_{20}^{\circ}=0$ represents the phase of maximum energy gain.

maximized energy gain, the phase jitter is significantly diminished by bimodal gun. Around the points of $d t / d \phi_{10}=0$, where the local minimum of flight time locates at, the slope of flight time as function of injection phase for bimodal mode is also lower than that of single mode, indicating that the low phase jitter injection phase range in bimodal gun is wider.

Figure 7(b) shows the slope of flight time as function of harmonic mode injection phase $d t / d \phi_{20}$ in a $4^{\circ}$ range around the maximum energy gain. $\triangle \phi_{20}$ represents the C-band nominal phase distance away from the maximum energy gain. The point of $\triangle \phi_{20}=0$ represents the maximum energy gain. Within a range of $6^{\circ}$, the slope of flight time as function of phase $d t / d \phi_{20}$ is lower than $10^{-10}$, indicating that the phase jitter of the 2 nd harmonic mode is negligible.

In conclusion, with the flat-top rf field in the cavity, the bimodal gun can compensate the phase jitter in the mentioned amplitude-jitter-free scheme, composing a low jitter UED facility that has the potential of breaking the existing time resolution barrier in MeV UED.

\section{MECHANICAL CONSIDERATION}

The detailed rf and mechanical engineering designs have been carried out. The cavity, the mode launcher and the directional coupler are three parts that can be manufactured separately, allowing multiple iterations and tuning as needed. The bimodal assembly is shown in Fig. 8.

Considering tuning and diagnosis, knobs and ports are added to the cavity. To tune the cavity precisely, three sets of deformation tuning spots are located in different areas around the cavity. The two tilted ports added in the 3D simulations in Fig. 1 are for diagnosing fields and other conditions within the cavity. With a $45^{\circ}$ angle tilted, one of the ports can be used as the window for observing the condition of the cathode.

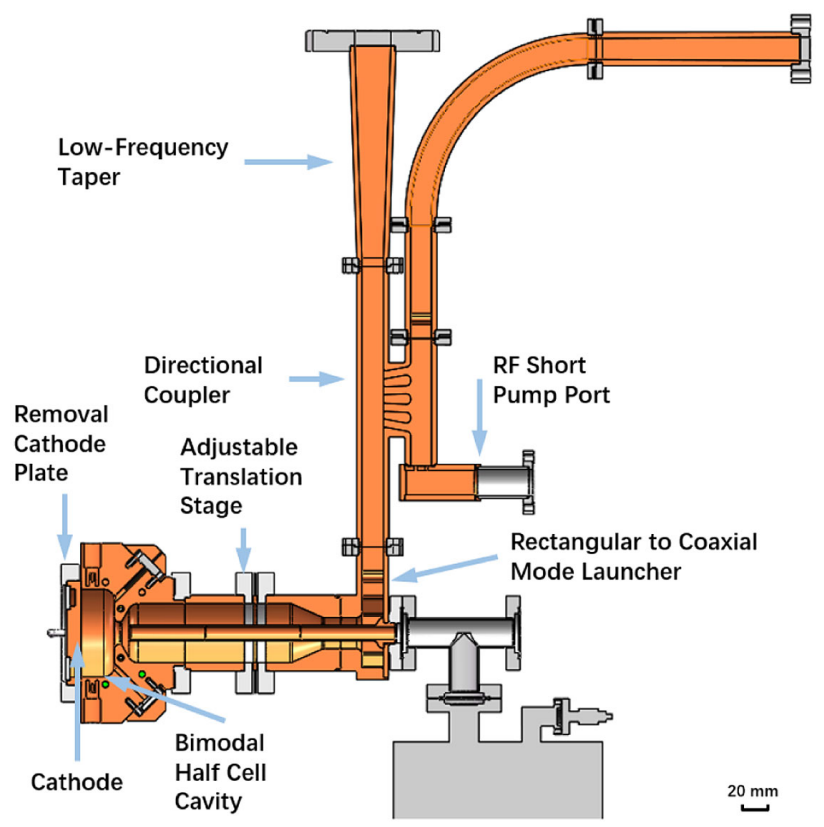

FIG. 8. Engineering drawing of the bimodal gun.

The cavity can be assembled without brazing. A loop of indium wire is used to obtain the vacuum seal and the perfect $\mathrm{rf}$ contact. When the cathode plate is clamped to the main part of the cavity, a soft indium wire in between the two parts provides the vacuum seal [30]. The nonbrazed cavity has two advantages over brazed cavity. First, without brazing, the oxygen-free copper can retain a high hardness [31]. Second, the removable back wall with the cathode assembly provides a simple modifiable platform for testing different cathode designs, such as $\mathrm{Cu}$ cathode, or possibly a gridded triode cathode [32,33].

To modify the coupling factor of the cavity, inspired by [34], an adjustable translation stage is inserted in the middle of the coaxial waveguide. Adjusting the thickness of the copper gasket which is clamped by the two symmetrical counterflanges can change the distance between the cathode and the tip of the inner metal cylinder, thus the coupling factor of both rf modes are adjusted.

\section{FUTURE STUDY}

There are potential alternates not being detailed here. Rather, they are listed in this section to make the point that research and design proposed here can open the door to a rich variety of future developments to benefit the accelerator and light source technology.

Further analysis should be performed on the pulsed heating and multipaction issue. The two-frequency superposition allows for the suppression of field emission and secondary electron emission [35] in the bimodal gun cavity, where the rf electric field pointing into one wall is significantly smaller than the field pointing out of the wall. A strong pointing-out-of-wall field will raise the 
effective work function barrier to suppress the field emission and secondary electron emission. Furthermore, due to the quadratic dependence on the ratio of the two mode amplitudes, the temperature rise due to pulsed heating can also be lessened [36]. As mentioned in Sec. II, due to the much lower phase velocity of C-band in the bimodal gun, the advantage and the beam deceleration of the 1.5-cell bimodal gun should be investigated from the beam dynamics point of view in a future study.

\section{CONCLUSION}

The design of a bimodal gun operating at S-band and C-band was developed in a collaboration between Wright Laboratory, Department of Physics, Yale University and Shanghai Advanced Research Institute, Chinese Academy of Sciences.

The confirmation of theory and simulation indicate that a frequency combination with lower harmonic frequency like S- and C-band, rather than S- and X-band (1st and 3rd), poses less budget for rf source, and can achieve the similar beam dynamics benefits. Good rf performance is obtained in simulation with a novel design of the directional coupler and mode launcher. Beam dynamic simulations in this paper show that the bimodal gun has the advantages of suppressing emittance growth and energy spread in $\mathrm{MeV}$ UEM, which can enhance the spatial resolution significantly. Suppression of timing jitter in MeV UED has also been observed in simulations, which has the potential of breaking the existing time resolution barrier in corporation with a DBA compression scheme.

With the aim of producing a prototype, further engineering and fabrication are underway at Shanghai Advanced Research Institute. The next steps in the development of this device will include a systematic study of rf tolerances, thermomechanical effects and beam stability.

\section{ACKNOWLEDGMENTS}

The author would like to thank Mikhael Guy from Yale Center for Research Computing for helpful technical support on high performance computing (HPC) at Yale. This work was supported by U.S. Department of Energy, under Award No. DE-SC00015158, and National Natural Science Foundation of China (No. 11675249).

[1] X. Wang, P. Musumeci, E. Lessner, and J. Goldstein, Report of the Basic Energy Sciences Workshop on the Future of Electron Sources, September 8-9, 2016, Tech. Rep. (USDOE Office of Science (SC), 2016).

[2] D. Xiang, F. Fu, J. Zhang, X. Huang, L. Wang, X. Wang, and W. Wan, Accelerator-based single-shot ultrafast transmission electron microscope with picosecond temporal resolution and nanometer spatial resolution, Nucl. Instrum. Methods Phys. Res., Sect. A 759, 74 (2014).
[3] C. Lu, T. Jiang, S. Liu, R. Wang, L. Zhao, P. Zhu, Y. Liu, J. $\mathrm{Xu}, \mathrm{D} . \mathrm{Yu}, \mathrm{W}$. Wan et al., Imaging nanoscale spatial modulation of a relativistic electron beam with a mev ultrafast electron microscope, Appl. Phys. Lett. 112, 113102 (2018).

[4] X. J. Wang, D. Xiang, T. K. Kim, and H. Ihee, Potential of femtosecond electron diffraction using near-relativistic electrons from a photocathode rf electron gun, J. Korean Phys. Soc. 48, 390 (2006), https://www.researchgate .net/publication/236512432_Potential_of_Femtosecond_ Electron_D iffraction_Using_Near- Relativistic_Electrons_ from_a_Photocathode_RF_Electron_Gun\#fullTextFileContent.

[5] K.-J. Kim, Rf and space-charge effects in laser-driven rf electron guns, Nucl. Instrum. Methods Phys. Res., Sect. A 275, 201 (1989).

[6] D. Dowell, Sources of emittance in $\mathrm{rf}$ photocathode injectors: Intrinsic emittance, space charge forces due to non-uniformities, rf and solenoid effects, arXiv:1610 $.01242 \mathrm{v} 3$.

[7] L. Serafini, R. Rivolta, and C. Pagani, Neutralization of the emittance blowup induced by rf time dependent forces in rf guns, Nucl. Instrum. Methods Phys. Res., Sect. A 318, 301 (1992).

[8] Z. T. Zhao and Y.Z. Lin, Beam dynamics characteristic in $1 / 2+m$ cell's harmonic microwave gun, High Energy Phys. Nucl. Phys. 18, 664 (1994).

[9] D. H. Dowell, M. Ferrario, T. Kimura, J. W. Lewellen, C. Limborg, P. Raimondi, J. F. Schmerge, L. Serafini, T. I. Smith, and L. M. Young, A two-frequency rf photocathode gun, Nucl. Instrum. Methods Phys. Res., Sect. A 528, 316 (2004).

[10] J. W. Lewellen and J. Noonan, Field-emission cathode gating for rf electron guns, Phys. Rev. Accel. Beams 8, 033502 (2005).

[11] J. Raguin, R. Bakker, K. Li, M. Pedrozzi et al., A twofrequency rf cavity for the psi low emittance gun, in Proceedings of the 27th International Free Electron Laser Conference (JACoW, Stanford, California, 2005), pp. 324-327, https://www.researchgate.net/publication/ 273447075_A_Two-Frequency_RF_Cavity_for_the_PSI_ Low_Emittance_Gun.

[12] D. Li, W. C. Fang, Q. Gu, Z. Wang, M. Zhang, J. H. Tan, C. P. Wang, and Z. T. Zhao, Simulation studies on twofrequency rf gun, Nuclear Science and Techniques, 25, 040101 (2014).

[13] R. Li and P. Musumeci, Single-shot mev transmission electron microscopy with picosecond temporal resolution, Phys. Rev. Applied 2, 024003 (2014).

[14] Z. Zhang, H. Qian, and C.-X. Tang, A spatially separated two frequency rf gun design for beam brightness improvement, in Proc. of International Particle Accelerator Conference (IPAC'16), Busan, Korea, May 8-13, 2016, 7 (JACoW, Geneva, 2016), pp. 2572-2575.

[15] P. Musumeci, J. G. Navarro, J. Rosenzweig, L. Cultrera, I. Bazarov, J. Maxson, S. Karkare, and H. Padmore, Advances in bright electron sources, Nucl. Instrum. Methods Phys. Res., Sect. A 907, 209 (2018).

[16] Y. Jiang, L. Wang, S. Shchelkunov, W. Fang, and J. Hirshfield, Bimodal electron gun r\&d, in IEEE Advanced 
Accelerator Concepts Workshop (AAC) (IEEE, Breckenridge, CO, 2018), pp. 1-5, https://ieeexplore.ieee.org/ document/8659391.

[17] L. Wang, W. Fang, J. Hirshfield, Y. Jiang, S. Shchelkunov, and Z. Zhao, 1st+2nd Harmonic Photocathode Bimodal Gun R\&D, in Proc. 10th International Particle Accelerator Conference (IPAC'19), Melbourne, Australia, 19-24 May 2019, 10 (JACoW, Geneva, 2019), pp. 2054-2057.

[18] K. T. McDonald, Design of the laser-driven rf electron gun for the bnl accelerator test facility, IEEE Trans. Electron Devices 35, 2052 (1988).

[19] Cst studio suite, computer simulation technology (cst), https://www.cst.com/products/csts2.

[20] L. Serafini, Beam dynamics in rf guns and emittance correction techniques, Nucl. Instrum. Methods Phys. Res., Sect. A 340, 40 (1994).

[21] I. Isaev and M. Krasilnikov, Rf field asymmetry simulations for the pitz rf photo gun, DPG 2015, Wuppertal, Germany (2015), https://bib-pubdb1.desy.de/record/ 291696/files/.

[22] J. Reed, The multiple branch waveguide coupler, IRE Trans. Microwave Theory Techniques 6, 398 (1958), https://ieeexplore.ieee.org/document/1125213.

[23] K. Flottmann, Astra: A space charge tracking algorithm, http://www.desy.de/mpyflo.

[24] J. E. Clendenin, Rf photoinjectors, in Proceedings of the 1996 Linear Accelerator Conference, Geneva, Switzerland (CERN, Geneva, 1999), pp. 298-302.

[25] C. Travier, An introduction to photo-injector design, Nucl. Instrum. Methods Phys. Res., Sect. A 340, 26 (1994).

[26] L. Serafini and J. B. Rosenzweig, Envelope analysis of intense relativistic quasilaminar beams in rf photoinjectors: Ma theory of emittance compensation, Phys. Rev. E 55, 7565 (1997).
[27] Poisson superfish, http://laacg.lanl.gov/laacg/services/ download_sf.phtml.

[28] M. Reiser and P. O'Shea, Theory and Design of Charged Particle Beams (Wiley Online Library, New York, 1994), Vol. 312.

[29] F. Qi, Z. Ma, L. Zhao, Y. Cheng, W. Jiang, C. Lu, T. Jiang, D. Qian, Z. Wang, W. Zhang, P. Zhu, X. Zou, W. Wan, D. Xiang, and J. Zhang, Breaking 50 Femtosecond Resolution Barrier in Mev Ultrafast Electron Diffraction with a Double Bend Achromat Compressor, Phys. Rev. Lett. 124, 134803 (2020).

[30] T. Bernat, D. Blair, and B. Hymel, Extrusion of indium wire for vacuum seals, Rev. Sci. Instrum. 46, 225 (1975).

[31] S. I. Wright and F. Heidelbach, Microtextural characterization of annealed and deformed copper, Mater. Sci. Forum 157-162, 1313 (1994).

[32] S. Nagaitsev, O. Nezhevenko, V. Yakovlev, R. Andrews, N. Solyak, A. Lunin, D. Sun, and M. Church, L-band rf gun with a thermionic cathode, in Proceedings of the 11th European Particle Accelerator Conference, Genoa, 2008 (EPS-AG, Genoa, Italy, 2008), p. MOPP076.

[33] K. Masuda, T. Shiiyama, T. Kii, H. Ohgaki, K. Kanno, and E. Tanabe, Development of a thermionic triode rf gun, in Proceedings of the 31st International Free Electron Laser Conference (FEL 09), Liverpool, UK (STFC Daresbury Laboratory, Warrington, 2009), pp. 281-284.

[34] I. Isaev, Stability and performance studies of the PITZ photoelectron gun, Ph.D. thesis, Universitt Hamburg, 2017.

[35] S. Kuzikov, S. Y. Kazakov, Y. Jiang, and J. Hirshfield, Asymmetric Bimodal Accelerator Cavity for Raising rf Breakdown Thresholds, Phys. Rev. Lett. 104, 214801 (2010).

[36] Y. Jiang and J. Hirshfield, Multi-harmonic accelerating cavities for rf breakdown studies, Proceedings of PAC2013, Pasadena, CA USA (2013), https://accelconf .web.cern.ch/pac2013/papers/wepma28.pdf. 